\title{
Correction to: Antimicrobial activity and DNA/HSA interaction of fluorinated 3,6,9-trisubstituted acridines
}

Patrik Nunhart ${ }^{1}$ - Eva Konkolová ${ }^{1}$ - Ladislav Janovec ${ }^{2}$. Jana Kašpárková ${ }^{3}$ - Jaroslav Malina ${ }^{3}$. Viktor Brabec ${ }^{3}$. Mária Matejová ${ }^{2} \cdot$ Beata Miltáková $^{2}$. Ladislav Novotny ${ }^{4}$ Oludotun A. Phillips ${ }^{4} \cdot$ Edet Udo $^{5}$ • Mária Kozurkova ${ }^{1,6}$

Published online: 17 February 2020

(c) Institute of Chemistry, Slovak Academy of Sciences 2020

\section{Correction to: Chemical Papers}

https://doi.org/10.1007/s11696-020-01079-4

An Online First version of this article was made available online at https://link.springer.com/article/10.1007/s1169 6-020-01079-4 on 3 February 2020.

Unfortunately the incorrect figure has been published in the article. The corrected figure is shown below.

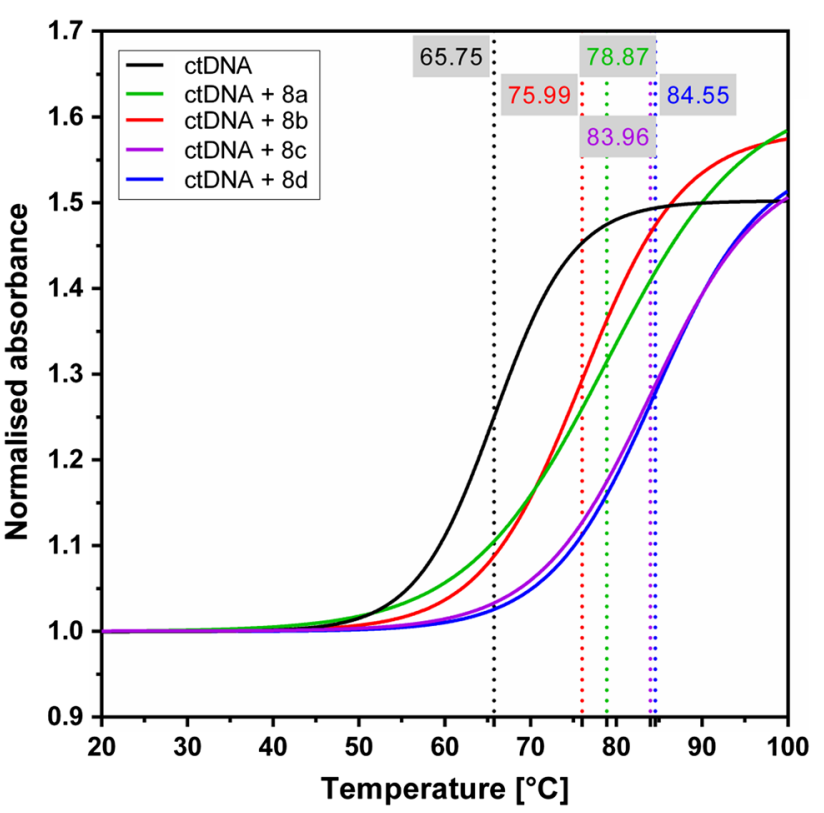

The original article can be found online at https://doi.org/10.1007/ s11696-020-01079-4.

Mária Kozurkova

maria.kozurkova@upjs.sk

1 Department of Biochemistry, Institute of Chemistry, Faculty of Science, P. J. Šafárik University in Košice, Šrobárova 2, 04154 Košice, Slovak Republic

2 Department of Organic Chemistry, Institute of Chemistry, Faculty of Science, P. J. Šafárik University in Košice, Šrobárova 2, 04154 Košice, Slovak Republic

3 Department of Biophysics, Faculty of Science, Palacky University in Olomouc, Olomouc, Czech Republic

4 Faculty of Pharmacy, Department of Pharmaceutical Chemistry, Kuwait University, Kuwait City, Kuwait

5 Faculty of Medicine, Department of Microbiology, Kuwait University, Kuwait City, Kuwait

6 Biomedical Research Center, University Hospital Hradec Kralove, Sokolovska 581, 50005 Hradec Kralove,

Czech Republic 